\title{
More complexity to the Bloom's syndrome complex
}

\author{
Yilun Liu ${ }^{1}$ and Stephen C. West ${ }^{2,3}$ \\ ${ }^{1}$ Department of Therapeutic Radiology, Yale University School of Medicine, New Haven, Connecticut 06510, USA; ${ }^{2}$ London \\ Research Institute, Cancer Research UK, Clare Hall Laboratories, South Mimms EN6 3LD, United Kingdom
}

\begin{abstract}
Bloom's syndrome is caused by mutations in the $B L M$ gene. The $B L M$ gene product, BLM helicase, forms a complex with two other proteins, DNA topoisomerase III $\alpha$ and RMI1. In this issue of Genes \& Development, Wang and colleagues (pp. 2843-2855) and Meetei and colleagues (pp. 2856-2868) report the discovery of a fourth component of this complex called RMI2. RMI2 may be a representative of a new family of OBfold-containing proteins that are important for complex stabilization and checkpoint response.
\end{abstract}

In humans, mutations in the $B L M$ gene give rise to Bloom's syndrome (BS) (Ellis et al. 1995), a rare genetic disorder that is most prevalent in the eastern European Ashkenaki Jewish population (incidence 1/50,000). The prominent physical features associated with BS include sun-sensitive skin lesions, small stature, male infertility, and a susceptibility to infections and diabetes (German 1993). Most importantly, BS patients develop various types of cancers often at a young age (for more information about the disease, visit the Bloom's Syndrome Foundation Web site at http://www.bloomssyndrome. org). Cells derived from individuals with BS exhibit genome instability, the hallmark feature of which is an elevated frequency of sister chromatid exchange (SCE). Since this debilitating genetic disorder was first reported more than five decades ago (Bloom 1954), significant effort has been exerted to try to understand the precise molecular defects associated with BS. As a consequence, we now have a good understanding of the cellular actions and activities of the BLM protein (Wu and Hickson 2006).

\section{The human RECQ family helicases}

BLM is a member of the RecQ family of DNA helicases (Ellis et al. 1995). The family is named after the Escherichia coli ortholog $\operatorname{RecQ}$, which is required for the

[Keywords: Bloom's syndrome; BLM; RMI1/2; RECQ; double Holliday junction; topoisomerase III $\alpha$ ]

${ }^{3}$ Corresponding author.

E-MAIL stephen.west@cancer.org.uk; FAX 44-1707-625-811.

Article is online at http://www.genesdev.org/cgi/doi/10.1101/gad.1732808 efficient induction of the DNA damage (SOS) response and suppression of illegitimate recombination leading to DNA rearrangements (Hanada et al. 1997). RecQ homologs are also present in Saccharomyces cerevisiae and Schizosaccharomyces pombe, where they are known as Sgs1 and Rqh1, respectively. Like RecQ, Sgs1 is required for the suppression of illegitimate recombination and SCEs, or crossovers, that arise by homologous recombination (Watt et al. 1996). Mutants of sgs1 show an increased sensitivity to agents that cause DNA damage or promote S-phase arrest, such as UV, hydroxyurea, and methylmethanesulfonate, indicating a role in DNA replication, recombination, and repair.

Perhaps surprisingly, although E. coli and yeast contain only one RecQ protein, there are no less than five members of this protein family in humans. They are known as RECQ1, BLM, WRN, RECQ4, and RECQ5, and all possess a domain structure to suggest that they are likely to function as DNA helicases. Indeed, RECQ1, BLM, WRN, and RECQ5 have been shown to unwind a variety of DNA substrates including replication forks, $3^{\prime}$ or $5^{\prime}$ flaps, and Holliday junctions (HJs), consistent with a role in DNA metabolism (Bachrati and Hickson 2003; Opresko et al. 2004). The one exception to this rule is RECQ4, for which it has not been possible to demonstrate helicase activity in vitro (Macris et al. 2006). In addition to the helicase activities, all five human RECQ proteins promote the reannealing of complementary ssDNAs (Bachrati and Hickson 2008).

Because the human RECQ proteins exhibit similar biochemical properties and are implicated in common DNA metabolic processes, it might be reasonable to think that these proteins have overlapping or redundant cellular functions. However, this is clearly not the case, as mutations in different RECQ proteins lead to distinct clinical syndromes (Hickson 2003). While mutations in BLM cause BS, mutations in WRN are linked to Werner Syndrome (WS), and RECQ4 is defective in RothmundThomson Syndrome (RTS). Moreover, while individuals with BS or RTS exhibit various physical and mental developmental abnormalities, the most striking phenotype associated with WS is premature aging. Most importantly, all these patients, in particular those with BS, 
have a high risk of cancer predisposition. As a consequence, cancer is the primary cause of death for BS patients before the age of 30 .

The most striking feature in cell lines derived from BS patients is the high levels of SCE accompanied with the formation of multi-radial chromosomes, which is a potential key factor leading to cell transformation and tumorigenesis. However, recent studies with recq5 $5^{-/-}$embryonic stem cells and primary embryonic fibroblasts derived from rec $q 5^{-/-}$knockout mice demonstrated similar phenotypes to those found associated with BS. The recq5 $5^{-/-} \mathrm{blm}^{-/-}$double mutants exhibited an even greater frequency of SCEs than either the rec $q 5^{-/-}$or the $b_{1 m^{-1-}}$ single mutants, suggesting that these two proteins suppress crossover formation using different mechanisms. Thus, BLM and RECQ5 play nonredundant roles in cancer prevention by regulating SCE formation and preventing chromosome aberration ( $\mathrm{Hu}$ et al. 2005, 2007).

Given that BLM and RECQ5 exhibit very similar biochemical properties, why do mutations in both of these proteins contribute to an elevated SCE frequency? To gain insight into the nonredundant roles of the RECQ family proteins, recent efforts have focused on identifying the specific protein-protein interactions of each of the RECQ helicases. Importantly, it was shown that RECQ5, but not the other RECQs, forms a stable complex with chromatin-associated RNA polymerase II (RNAPII) (Aygun et al. 2008; Izumikawa et al. 2008). The interaction of RECQ5 with RNAPII might indicate that RECQ5 suppresses SCE formation by specifically preventing recombination events associated with transcription. Similarly, the architecture of the BLM protein complex has been gradually established during the past 5 years. In this regard, the discovery of an additional component of the BLM complex, as described in this issue of Genes \& Development (Singh et al. 2008; Xu et al. 2008), may turn out to be significant. It is likely that further analysis of such specific protein-protein interactions will reveal the complex functions and regulatory aspects of BLM in human cells.

\section{The BLM core complex}

Over the past decade, many proteins have been described as potential BLM-interacting proteins, but it is now thought likely that several of them interact either transiently or indirectly with BLM. Only a few polypeptides are now considered as part of the true BLM core complex (Fig. 1), and these include topoisomerase III $\alpha$ (TopoIII $\alpha$ ), Replication Protein A (RPA), and RMI1. Functional interactions take place between BLM and TopoIII $\alpha$ (Wu et al. 2000; Wu and Hickson 2003) and are mimicked by similar associations that take place between their yeast homologs, Sgs1 and TopoIII (Gangloff et al. 1994; Bennett et al. 2000). The combined actions of BLM with TopoIII $\alpha$ leads to a remarkable biochemical reaction that results in the dissociation of DNA structures containing double HJs (dHJs), structures that represent intermediates in the process of homologous recombination (Wu and Hickson 2003). The product of the dissolution reaction is genetically silent in that the HJs are removed without any exchange of DNA between the interacting molecules (they are noncrossover products). Given that cells contain alternative pathways to resolve these intermediates, into both crossover and noncrossover products, it is now clear why the absence of BLM leads to an elevated frequency of SCEs. In essence, BLM-TopoIII $\alpha$ complex acts as a suppressor of crossing over and SCE formation.

In contrast to TopoIII $\alpha$, which interacts specifically with BLM, RPA interacts with several RECQ family members (Bachrati and Hickson 2008). RPA is an oligonucleotide-binding, or OB-fold, containing protein and is functionally similar to the $E$. coli single-stranded binding protein SSB. However, whereas SSB consists of a single polypeptide, RPA is comprised of three polypeptides associated together via their OB-fold domains. The
Figure 1. Schematic diagram indicating the BLM core complex and a variety of possible subcomplexes that are thought to exist in human cells. Direct interactions between two proteins are indicated by solid black double arrows, whereas potential interactions are shown with dashed red double arrows.

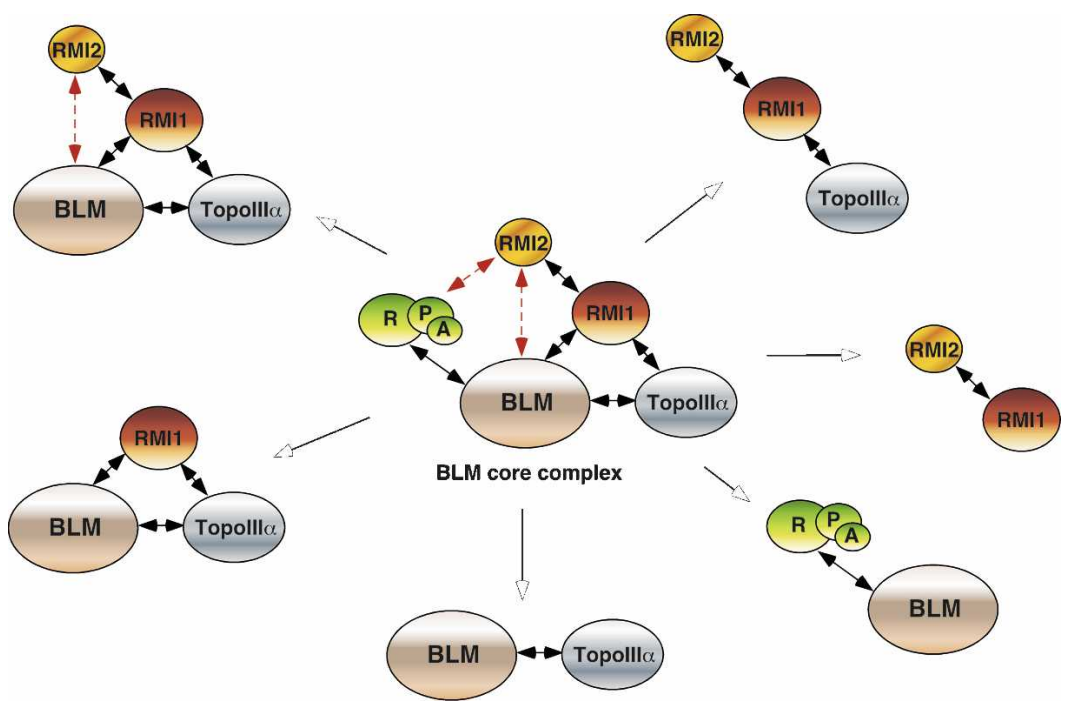


functional association of RPA with all RECQ helicases results in a stimulation of DNA unwinding, which occurs by two distinct mechanisms: (1) stabilization of the ssDNA that results from DNA unwinding, and (2) direct protein-protein interactions. Since RECQ helicases exhibit a strand-annealing activity in addition to their ATP-dependent DNA helicase activities, RPA appears capable of counteracting DNA annealing by binding and stabilizing the ssDNA products. These reactions, however, are thought to require specific protein-protein interactions since $E$. coli SSB cannot substitute for RPA to efficiently stimulate human RECQ helicases (Bachrati and Hickson 2008).

In 2005, another member of the BLM core complex, RMI1 (originally called BLAP75), was identified as a component of the BLM complex purified from human cells (Yin et al. 2005) and also as part of the yeast Sgs1TopoIII complex (Chang et al. 2005; Mullen et al. 2005). Interestingly, RMIl's association with BLM appears to be accomplished by independent direct physical interactions with both BLM and TopoIII $\alpha$ (Raynard et al. 2006; Chen and Brill 2007), although it is not clear whether the interactions are mutually exclusive as the BLM and TopoIII $\alpha$ interaction domains on RMI1 map to the same $\mathrm{N}$-terminal region (Raynard et al. 2008). Nevertheless, it has been shown that RMI1 plays an important role in stimulating $\mathrm{dHJ}$ dissolution catalyzed by BLM-TopoIII $\alpha$ (Wu et al. 2006; Raynard et al. 2008). Recent results show that TopoIII $\alpha$-RMIl complex also stimulates the movement or branch migration activity of BLM on single HJs, and it is thought likely that RMI1 interacts primarily with TopoIII $\alpha$ within this complex (Raynard et al. 2008).

RMIl contains two OB-fold domains and is capable of binding to ssDNA, dsDNA, or more complex DNA structures such as dHJs (Wu et al. 2006; Raynard et al. 2008). Intriguingly, unlike RPA, the DNA-binding activity of RMIl is dispensable for the stimulation of BLMdependent helicase activity. Thus, in contrast to the RPA-dependent stimulation that occurs through both DNA-protein and protein-protein interactions, RMI1 affects BLM function only through specific protein-protein contacts. This is an interesting difference as it suggests that the OB-fold domain proteins RPA and RMI exhibit functionally distinct mechanisms of stimulation.

\section{RMI2, a new OB domain protein}

The association of two OB-fold proteins, RPA and RMI1, with BLM suggests that other OB-fold proteins might interact with BLM using this common domain. This possibility leads us to the two papers in this issue (Singh et al. 2008; Xu et al. 2008) that report the identification of a novel BLM complex-associated protein that has been called RMI2. The protein was found to be present in BLM or RMI1 immunoprecipitates and was subsequently identified as a $15.8-\mathrm{kDa}$ polypeptide by mass spectrometry (Singh et al. 2008; Xu et al. 2008). RMI1 was found to interact with RMI2 via the C-terminal OB-fold domain of RMI1 and the OB-fold domain of RMI2, remi- niscent of the interactions that occur between the largest subunits of RPA, RPA1, and RPA2. Purified recombinant RMI1 and RMI2 stably associate to form a heterodimeric complex that has greatly improved solubility compared with either of the two individual subunits. Indeed, it is possible that the RMI1/RMI2 heterodimer is a functional protein, reminiscent of the RPA heterotrimer (Fig. 1). This observation raises the question of whether RMI might actually be a paralog of RPA. However, this is clearly not the case because-in contrast to RPA, which exhibits a high affinity for DNA-the RMI complex actually fails to display DNA-binding activity. Since RMI1 alone has been shown to bind to DNA (Wu et al. 2006; Raynard et al. 2008), it seems likely that the interaction of RMI1 with RMI2 inhibits its ability to bind DNA. Interestingly, the DNA-binding domain of RMI1 has been mapped to the same region as the RMI2-interaction domain, suggesting that inhibition occurs by competition between DNA binding versus protein-protein interaction (Raynard et al. 2008; Xu et al. 2008).

The stable association of RMI1 with RMI2 prompted the Wang group (Xu et al. 2008) and the Meetei group (Singh et al. 2008) to analyze the effects of the heterodimer on the ability of BLM-TopoIII $\alpha$ to promote $\mathrm{dHJ}$ dissolution. If RMI2 was expected to greatly stimulate $\mathrm{dHJ}$ dissolution, then the authors would have been very disappointed, as little significant effect was observed. Indeed, the small amount of stimulation seen by the Meetei group (Singh et al. 2008) may well be due simply to the enhanced stability of the RMI complex compared with RMI1 alone.

\section{RMI2 may be required for a subset of BLM reactions}

Homologous recombination provides an important cellular mechanism to repair double-strand breaks caused by DNA-damaging agents. Normally SCE formation by homologous recombination is low, most probably due to the $\mathrm{dHJ}$ dissolution activity of the BLM complex (Wu and Hickson 2003). Similarly, spontaneous SCE formation, which could occur during the repair of stalled replication forks, is also suppressed (Kuzminov 2001; Cox 2002). In a normal cell, there are several alternative mechanisms to resume replication at the stalled replication fork, and it is thought that BLM complex may act in multiple pathways that ultimately lead to fork recovery without crossover formation (Fig. 2). In some pathways of fork repair, the DNA helicase activity of BLM alone might be sufficient for recovery, possibly by the promotion of fork regression, while other pathways such as those involving $\mathrm{dHJs}$ require the presence of a more complete BLM complex containing TopoIII $\alpha$ and possibly the RMI proteins.

\section{RMI2, the stabilizer of the BLM core complex}

While the full spectrum of the potential biochemical functions of RMI2 requires further investigation, it seems likely that RMI2 may not serve primarily as a 
Figure 2. Potential roles of BLM and its interaction partners in human cells Schematic diagram indicating alternative pathways for the repair of stalled replication forks that arise as a result of DNA damage. Leading and lagging strand synthesis is shown in green and blue, respectively. $(a-c)$ Following fork blockage, RAD51 recombinase can promote strand exchange between paired sisters, such that the DNA synthesis can bypass the damage in the leading strand by use of the sister chromatid. $(c, d)$ The resulting recombination intermediate may then be dissociated by BLM-TopoIII $\alpha$ to give rise to a noncrossover product. (e) Alternatively, BLM could promote fork regression to produce a chicken foot structure, or HJ, with the newly synthesized strands annealed with each other. $(f)$ The newly synthesized strand from the undamaged parental strand can then serve as a template for lesion bypass. (d) The HJ may then be dissociated back to a replication fork allowing replication to continue. $(g)$ A further possibility is that the $5^{\prime}$-end of the newly synthesized single-strand of DNA could fold back and reanneal to its original parental strand. DNA synthesis and branch migration could also give rise to dHJs $(h)$, which in turn can be dissociated by BLM complex back to a replication fork structure without crossover formation $(d)$. On the other hand, in the absence of a functional BLM complex, HJs formed by either fork regression $(e)$ or by RAD51-dependent strand exchange reactions $(c)$ could be resolved by $\mathrm{HJ}$ resolvases leading to DSB formation, which in turn can be repaired by homologous recombination to restore the replication fork with crossover (i). The involvement of BLM in each pathway is indicated, as are the potential roles of BLM interacting proteins (red). In some cases, the roles of BLM-interacting proteins are unknown (gray).

catalytic component of the BLM core complex. Indeed, current evidence suggests that the function of RMI2 is to act as a stabilization factor for the BLM core complex in vivo. Using RNAi oligos to independently knock down each of the BLM core complex components, it was shown that the cellular stabilities of RMI1, RMI2, and TopoIII $\alpha$ proteins were dependent on each other (Singh et al. 2008; Xu et al. 2008). Indeed, the loss of any one protein greatly reduced the protein levels of the other two factors. In contrast, however, the protein levels of BLM remained unchanged; nor was BLM required for the stability of the RMI1, RMI2, or TopoIII $\alpha$ proteins. One possibility is that RMI1-RMI2-TopoIII $\alpha$ forms a stable heterotrimer, which in turn interacts with BLM to form the BLM core complex (Fig. 1). Consistent with this proposal, $\mathrm{Xu}$ et al. (2008) observed the RMI1-RMI2TopoIII $\alpha$ complex in cell-free extracts. Since RMIl interacts with both RMI2 and TopoIII $\alpha$, it is to be expected that RMI1 knockdown would affect the stability of RMI2 and TopoIII $\alpha$. At the present time, however, it is not clear whether RMI2 interacts directly with TopoIII $\alpha$, and it is perhaps more likely that TopoIII $\alpha$ and RMI2 affect each other indirectly through RMI1. The presence of a RMI1-RMI2-TopoIII $\alpha$ subcomplex raises the question of whether this complex might have a cellular role that is independent of BLM. It also opens the possibility that BLM might be stabilized through interactions with alternative protein factors in the absence of the RMI1RMI2-TopoIII $\alpha$ subcomplex.

In addition to its stabilization role, studies with a mutant form of RMI2 ${ }^{\mathrm{K} 121 \mathrm{~A}}$ indicate a regulatory function for RMI2 in terms of defining the interaction of BLM with the RMI1-RMI2-TopoIII $\alpha$ subcomplex (Xu et al. 2008). Indeed, the K121A mutation resulted in a complete fail- ure to interact with BLM, and this occurred independently of either RMI1 or TopoIII $\alpha$ (while both of these proteins retained the ability to interact with the mu$\operatorname{tant}$ ). However, it remains puzzling why the interaction between BLM and the RMI1-RMI2 ${ }^{\mathrm{K} 121 \mathrm{~A}}$-TopoIII $\alpha$ subcomplex was completely abolished, since both RMIl and TopoIII $\alpha$ can interact independently with BLM. Precisely how the K121A mutation in RMI2 affects the interaction of BLM with RMI1-RMI2-TopoIII $\alpha$ subcomplex clearly needs further investigation.

\section{Beyond the BLM core complex}

While the biochemical contributions of RMI2 to biochemical reactions mediated by the BLM complex remain questionable, it is clear that the presence of RMI2 is critical for the stability of the BLM core complex. It is also likely that RMI2 serves as a mediator that links BLM with other factors important for DNA damage and cell cycle checkpoint response. Firstly, RMI2 was found to be important for the recruitment or stabilization of BLM complexes to sites of DNA damage, as measured by the formation of BLM and RMIl foci after DNA-damaging treatment. Of course, it is possible that the decrease in RMI1 focus formation in the RMI2-depleted cells is a direct result of the reduced concentration of RMIl protein. However, given that the protein levels of BLM do not decrease after RMI2 depletion, and yet the number of cells with BLM foci were reduced significantly, this is thought to be unlikely. Interestingly, RMI2-depleted cells show an elevated frequency of SCEs (Xu et al. 2008) and an increased number of chromosome breaks (Singh et al. 2008). One possible scenario is that the RMI1RMI2-TopoIII $\alpha$ complex recognizes sites of DNA dam- 
age and relocalizes BLM to these sites through proteinprotein interactions. This possibility is supported by observations indicating that RMI2 helps to target BLM to chromatin (Singh et al. 2008). However, since the RMI1RMI2 subcomplex shows little affinity with DNA, the interactions with DNA must be achieved through TopoIII $\alpha$. Alternatively, RMI1-RMI2-TopoIII $\alpha$ might act as a bridge that links BLM to other DNA damage response factors and subsequently facilitates the recruitment of BLM to the sites of repair.

In addition to DNA damage-induced focus formation, RMI2 also influences the mitotic phosphorylation of BLM (Singh et al. 2008). One possibility is that RMI2, in collaboration with RMI1 and TopoIII $\alpha$, mediates interactions between the mitotic checkpoint kinases and BLM. It has been shown that RMI2 itself is phosphorylated by the mitotic checkpoint kinases, but the physiological relevance of this phosphorylation event remains to be elucidated. With these issues in mind and the fact that BLM is a highly interactive protein that is capable of transient association with a number of proteins involving in different aspects of DNA metabolism, it will be interesting to determine whether RMI2 plays a specific role in either connecting or excluding BLM from other DNA repair or replication factors beyond those present within the BLM core complex.

\section{Acknowledgments}

Y.L. is supported by grants from the NIH, American Cancer Society, American Federation for Aging Research, and Breast Cancer Alliance. S.C.W. is supported by grants from Cancer Research UK, the Louis-Jeantet Foundation, and the EU DNA Repair Consortium.

\section{References}

Aygun, O., Svejstrup, J.Q., and Liu, Y. 2008. A RECQ5-RNA polymerase II association identified by targeted proteomic analysis of human chromatin. Proc. Natl. Acad. Sci. 105: 8580-8584.

Bachrati, C.Z. and Hickson, I.D. 2003. Helicases, suppressors of tumorigenesis and premature aging. Biochem. J. 374: 577606.

Bachrati, C.Z. and Hickson, I.D. 2008. RECQ helicases: Guardian angels of the DNA replication fork. Chromosoma 117: 219-233.

Bennett, R.J., Noirot-Gros, M.F., and Wang, J.C. 2000. Interaction between yeast Sgs1 helicase and DNA topoisomerase III. J. Biol. Chem. 275: 26898-26905.

Bloom, D. 1954. Congenital telangiectatic erythema resembling lupus erythematosus in dwarfs: Probably a syndrome entity. AMA Am. J. Dis. Child. 88: 754-758.

Chang, M., Bellaoui, M., Zhang, C.Y., Desai, R., Morozov, P., Delgado-Cruzata, L., Rothstein, R., Freyer, G.A., Boone, C., and Brown, G.W. 2005. RMI1/NCE4, a suppressor of genome instability, encodes a member of the RECQ helicase/Topo III complex. EMBO I. 24: 2024-2033.

Chen, C.F. and Brill, S.J. 2007. Binding and activation of DNA topoisomerase III by the RMI1 subunit. J. Biol. Chem. 282: 28971-28979.

Cox, M.M. 2002. The nonmutagenic repair of broken replica- tion forks via recombination. Mutat. Res. 510: 107-120.

Ellis, N.A., Groden, J., Ye, T.Z., Straughen, J., Lennon, D.J., Ciocci, S., Proytcheva, M., and German, J. 1995. The Blooms Syndrome gene product is homologous to RecQ helicases. Cell 83: 655-666.

Gangloff, S., McDonald, J.P., Bendixen, C., Arthur, L., and Rothstein, R. 1994. The yeast type-I topoisomerase Top3 interacts with Sgs1, a DNA helicase homolog-A potential eukaryotic reverse gyrase. Mol. Cell. Biol. 14: 83918398.

German, J. 1993. Bloom syndrome: A Mendelian prototype of somatic mutational disease. Medicine 72: 393-406.

Hanada, K., Ukita, T., Kohno, Y., Saito, K., Kato, J., and Ikeda, H. 1997. RecQ DNA helicase is a suppressor of illegitimate recombination in Escherichia coli. Proc. Natl. Acad. Sci. 94: 3860-3865.

Hickson, I.D. 2003. RECQ helicase: Caretakers of the genome. Nat. Rev. Mol. Cell Biol. 3: 169-178.

Hu, Y.D., Lu, X.C., Barnes, E., Yan, M., Lou, H., and Luo, G.B. 2005. RECQL5 and BLM RECQ DNA helicases have nonredundant roles in suppressing crossovers. Mol. Cell. Biol. 25: 3431-3442.

Hu, Y., Raynard, S., Sehorn, M.G., Lu, X., Bussen, W., Zheng, L., Stark, J.M., Barnes, E.L., Chi, P., Janscak, P., et al. 2007. RECQL5 helicase regulates homologous recombination and suppresses tumor formation via disruption of RAD51 presynaptic filaments. Genes \& Dev. 21: 30733084 .

Izumikawa, K., Yanagida, M., Hayano, T., Tachikawa, H., Komatsu, W., Shimamoto, A., Futami, K., Furiuichi, Y., Shinkawa, T., Yamauchi, Y., et al. 2008. Association of human DNA helicase RECQ5 $\beta$ with RNA polymerase II and its possible role in transcription. Biochem. J. 413: 505516.

Kuzminov, A. 2001. DNA replication meets genetic exchange: Chromosomal damage and its repair by homologous recombination. Proc. Natl. Acad. Sci. 98: 8461-8468.

Macris, M.A., Krejci, L., Bussen, W., Shimamoto, A., and Sung, P. 2006. Biochemical characterization of the RECQ4 protein, mutated in Rothmund-Thomson syndrome. DNA Repair (Amst.) 5: 172-180.

Mullen, J.R., Nallaseth, F.S., Lan, Y.Q., Slagle, C.E., and Brill, S.J. 2005. Yeast Rmil/Nce4 controls genome stability as a subunit of the Sgs1-Top3 complex. Mol. Cell. Biol. 25: 44764487.

Opresko, P.L., Cheng, W.H., and Bohr, V.A. 2004. Junction of RECQ helicase biochemistry and human disease. I. Biol. Chem. 279: 18099-18102.

Raynard, S., Bussen, W., and Sung, P. 2006. A double Holliday junction dissolvasome comprising BLM, Topoisomerase III $\alpha$, and BLAP75. J. Biol. Chem. 281: 13861-13864.

Raynard, S., Zhao, W.M., Bussen, W., Lu, L., Ding, Y.Y., Busygina, V., Meetei, A.R., and Sung, P. 2008. Functional role of BLAP75 in BLM-topoisomerase III $\alpha$-dependent Holliday junction processing. J. Biol. Chem. 283: 1570115708.

Singh, T.R., Ali, A.M., Busygina, V., Raynard, S., Fan, Q., Du, C.-H., Andreassen, P.R., Sung, P., and Meetei, A.R. 2008. BLAP18/RMI2, a novel OB-fold-containing protein, is an essential component of the Bloom helicase-double Holliday junction dissolvasome. Genes \& Dev. (this issue). doi: 10.1101/gad.1725108.

Watt, P.M., Hickson, I.D., Borts, R.H., and Louis, E.J. 1996. Sgs1, a homolog of the Bloom's and Werner's syndrome genes, is required for maintenance of genome stability in Saccharomyces cerevisiae. Genetics 144: 935-945. 


\section{Liu and West}

Wu, L. and Hickson, I.D. 2003. The Bloom's syndrome helicase suppresses crossing over during homologous recombination. Nature 426: 870-874.

Wu, L. and Hickson, I.D. 2006. DNA Helicases required for homologous recombination and repair of damaged replication forks. Annu. Rev. Genet. 40: 279-306.

Wu, L., Davies, S.L., North, P.S., Goulaouic, H., Riou, J.F., Turley, H., Gatter, K.C., and Hickson, I.D. 2000. The Bloom's syndrome gene product interacts with topoisomerase III. $J$. Biol. Chem. 275: 9636-9644.

Wu, L., Bachrati, C.Z., Ou, J., Xu, C., Yin, J.H., Chang, M., Wang, W., Li, L., Brown, G.W., and Hickson, I.D. 2006. BLAP75/RMI1 promotes the BLM-dependent dissolution of homologous recombination intermediates. Proc. Natl. Acad. Sci. 103: 4068-4073.

Xu, D., Guo, R., Sobeck, A., Bachrati, C.Z., Yang, J., Enomoto, T., Brown, G.W., Hoatlin, M.E., Hickson, I.D., and Wang, W. 2008. RMI, a new OB-fold complex essential for Bloom syndrome protein to maintain genome stability. Genes \& Dev. (this issue). doi: 10.1101/gad.1708608.

Yin, J.H., Sobeck, A., Xu, C., Meetei, A.R., Hoatlin, M., Li, L., and Wang, W. 2005. BLAP75, an essential component of Bloom's syndrome protein complexes that maintain genome integrity. $E M B O$ J. 24: 1465-1476. 


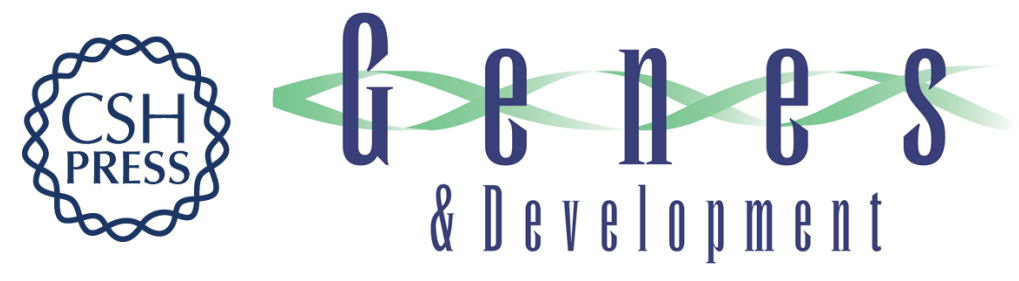

\section{More complexity to the Bloom's syndrome complex}

Yilun Liu and Stephen C. West

Genes Dev. 2008, 22:

Access the most recent version at doi:10.1101/gad.1732808

\section{Related Content RMI, a new OB-fold complex essential for Bloom syndrome protein to maintain genome stability \\ Dongyi Xu, Rong Guo, Alexandra Sobeck, et al. \\ Genes Dev. October, 2008 22: 2843-2855 BLAP18/RMI2, a novel OB-fold-containing protein, is an essential component of the Bloom helicasedouble Holliday junction dissolvasome \\ Thiyam Ramsing Singh, Abdullah Mahmood Ali, Valeria Busygina, et al. \\ Genes Dev. October, 2008 22: 2856-2868}

References This article cites 30 articles, 18 of which can be accessed free at:

http://genesdev.cshlp.org/content/22/20/2737.full.html\#ref-list-1

Articles cited in:

http://genesdev.cshlp.org/content/22/20/2737.full.html\#related-urls

\section{License}

Email Alerting

Receive free email alerts when new articles cite this article - sign up in the box at the top Service right corner of the article or click here.

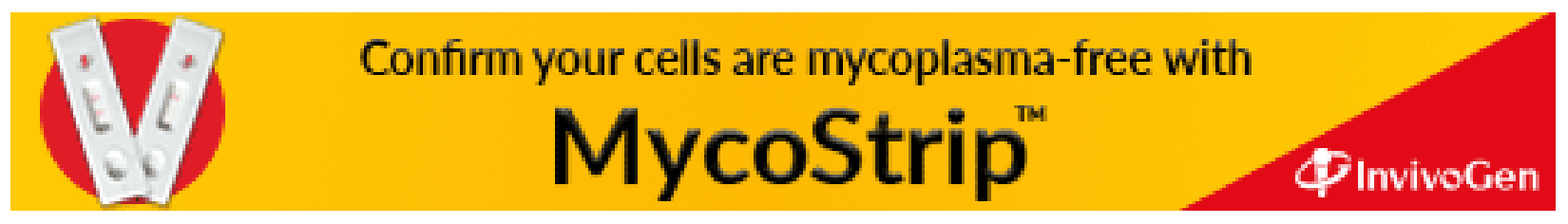

